\title{
Circulating miRNA expression over the course of colorectal cancer treatment
}

\author{
EVA KUDELOVA ${ }^{1}$, VERONIKA HOLUBEKOVA ${ }^{2}$, MARIAN GRENDAR ${ }^{2}$, ZUZANA KOLKOVA $^{2}$, \\ MAREK SAMEC $^{3}$, BARBORA VANOVA ${ }^{2}$, PETER MIKOLAJCIK ${ }^{1}$, MAREK SMOLAR $^{1}$, \\ ERIK KUDELA $^{3}$, LUDOVIT LACA ${ }^{1}$ and ZORA LASABOVA ${ }^{4}$ \\ ${ }^{1}$ Clinic of Surgery and Transplant Center, Jessenius Faculty of Medicine in Martin; ${ }^{2}$ Biomedical Center in Martin, \\ Jessenius Faculty of Medicine, Comenius University in Bratislava; ${ }^{3}$ Clinic of Gynecology and Obstetrics, \\ Jessenius Faculty of Medicine; ${ }^{4}$ Department of Molecular Biology and Genomics, \\ Jessenius Faculty of Medicine in Martin, Comenius University in Bratislava, Martin SK-03601, Slovak Republic
}

Received February 1, 2021; Accepted July 20, 2021

DOI: $10.3892 / \mathrm{ol} .2021 .13136$

\begin{abstract}
Colorectal cancer (CRC) is the third-most common cancer type in males and the second-most common cancer type in females, and has the second-highest overall mortality rate worldwide. Approximately $50 \%$ of patients in stage I-III develop metastases, mostly localized to the liver. All physiological conditions occurring in the organism are also reflected in the levels of circulating microRNAs (miRNAs/miRs) in patients. miRNAs are a class of small, non-coding, single-stranded RNAs consisting of 18-25 nucleotides, which have important roles in various cellular processes. The aim of the present study was to evaluate a panel of seven circulating miRNAs (miR-106a-5p, miR-210-5p, miR-155-5p, miR-21-5p, miR-103a-3p, miR-191-5p and miR-16-5p) as biomarkers for monitoring patients undergoing adjuvant treatment of CRC. Total RNA was extracted from the plasma of patients with CRC prior to surgery, in the early post-operative period $(n=60)$ and 3 months after surgery $(n=14)$. The levels of the selected circulating miRNAs were measured with the miRCURY LNA miRNA PCR system and fold changes were calculated using the standard $\Delta \Delta \mathrm{Cq}$ method. DIANA-miRPath analysis was used to evaluate the role of significantly deregulated miRNAs. The results indicated significant upregulation of miR-155-5p, miR-21-5p and miR-191-5p, and downregulation of miR-16-5p directly after the surgery. In paired follow-up samples, the most significant upregulation was detected for miR-106a-5p and $\mathrm{miR}-16-5 \mathrm{p}$, and the most significant downregulation was for miR-21-5p. Pathway analysis outlined the role of the
\end{abstract}

Correspondence to: Dr Veronika Holubekova, Biomedical Center in Martin, Jessenius Faculty of Medicine, Comenius University in Bratislava, 4C Malá Hora, Martin SK-03601, Slovak Republic E-mail: veronika.holubekova@uniba.sk

Key words: colorectal cancer, miRNA expression, pathway analysis, intestinal wound healing differentially expressed miRNAs in cancer development, but the same pathways are also involved in wound healing and regeneration of intestinal epithelium. It may be suggested that these processes should also be considered in studies investigating sensitive and easily detectable circulating biomarkers for recurrence in patients.

\section{Introduction}

Colorectal cancer (CRC) is the third-most common cancer type in males and the second-most common cancer type in females, and has the second-highest overall mortality rate worldwide (1). Major risk factors for CRC include a Western dietary pattern, sedentary lifestyle and increasing obesity due to lifestyle factors related to economic growth and industrialization. CRC has a relatively higher incidence in economically developed countries, which may be associated with a higher life expectancy, education and human developmental index due to better diagnosis of CRC (2). Furthermore, preventable lifestyle habits such as smoking, alcohol consumption and obesity contribute to an increased risk of tumour formation (3). Abdominal fat is sub-divided into two compartments: Visceral adipose tissue (VAT) and subcutaneous adipose tissue. VAT is responsible for the secretion of pro-inflammatory adipokines (such as TNF); in addition, it is infiltrated with macrophages, leading to chronic inflammation and promoting tumour growth (4). Furthermore, insulin resistance related to obesity has an important role in the promotion of carcinogenesis. Chronic inflammation is also a condition responsible for the elevated risk of CRC in patients with ulcerative colitis and Crohn's disease (3). In general, cancer is a disease associated with ageing; the risk of CRC markedly increases after the age of years 50. However, a higher incidence in individuals aged $<40$ years has also been observed (4).

Approximately $50 \%$ of patients in stage I-III develop metastases mostly localized to the liver (5); furthermore, $20 \%$ of patients already have liver metastases at the time-point of diagnosis (6). Patients with stage I disease have a 5-year survival rate of $90 \%$; however, a marked decline is observed 
in patients with stage IV disease (6). Understanding molecular pathway abnormalities is crucial for improving the diagnosis, prognosis and treatment of CRC, as it is a heterogeneous multifactorial disease with a range of different prognoses and responses to therapy (7). During the last decade, the development of novel targeted therapies and chemotherapeutics has led to improved clinical outcomes for patients with advanced metastatic disease. Despite the current screening methods and prognostic factors, there are still numerous patients that are not profiting from novel treatment strategies (4), which makes the early diagnosis of CRC recurrence key for improving prognosis and reducing mortality. Therefore, there is a significant requirement for a representative, sensitive and easily detectable biomarker (8).

MicroRNAs (miRNAs/miRs) are a class of small, non-coding, single-stranded RNAs consisting of approximately 18-25 nucleotides (9). They regulate the translation of protein-coding genes at the post-transcriptional level by binding to complementary sequences in the 3-untranslated regions of their target mRNAs (10), leading to inhibition of translation or degradation of the mRNA (11). In addition to tumour tissue, miRNAs have been identified in numerous biological sample types, including plasma, serum, saliva, faeces, urine, cerebrospinal and amniotic fluid (12). Circulating miRNAs are actively released into the bloodstream from tumour cells and may be detected in the form of exosomes, free or bound to proteins (11). miRNAs may either function as tumour suppressors through inhibition of oncogene expression or as oncogenic miRNAs by inhibiting the expression of tumour suppressor genes. Due to their localization within fragile sites in the genome, the expression of miRNAs may be dysregulated by different genetic alterations, including deletions, amplifications, translocations and point mutations, and also DNA hyper-methylation and hypo-methylation (10). Altered miRNA expression has been identified in various types of cancer, e.g., pancreatic cancer, hepatocellular cancer, breast cancer, CRC and lung cancer (13). miRNAs have important roles in cellular processes and are also involved in mechanisms of cancer development, such as cell proliferation, differentiation, apoptosis, angiogenesis and epithelial-mesenchymal transition. Several miRNAs have been suggested as candidates for CRC diagnosis; however, it is still difficult to draw clear conclusions (12).

Carcinoembryonic antigen (CEA) is a widely used marker for $\mathrm{CRC}$ recurrence detection recommended by the European Group on Tumour Markers and the American Society of Clinical Oncology $(14,15)$. Furthermore, CEA may be detected from peripheral venous blood, which makes CEA a suitable marker; however, its sensitivity is not sufficient in certain cases (16). The plasma level of CEA should be established pre-operatively to monitor the dynamics of its concentration after surgery, which is not always accomplished. In addition, CEA may only be present at low concentrations in patients with poorly differentiated tumours. miRNAs are easily detectable and stable biomarkers; however, further research is required to establish them as reliable biomarkers for local and distant recurrence of CRC. Implementation of miRNAs in clinical practice remains a challenge due to their diversity in affecting numerous molecular and cellular processes (17).
Apart from cancer, miRNAs have important roles in numerous molecular networks associated with inflammatory and autoimmune disorders. There is evidence that a connection exists between cancer and chronic inflammation (18), in which miR-155, miR-210 and miR-21 are involved. It has been indicated that upregulation of miR-155 results from oncogenic and inflammatory stimuli and triggers malignant transformation. Furthermore, miR-155 overexpression is frequently associated with high cytokine production (19). Studies have indicated that miR-21 is involved in both negative and positive feedback loops controlling inflammation. This has been demonstrated in different cell types under different conditions; therefore, this may be an explanation for the opposing effects of miR-21 on inflammation (20). Inflammation itself is linked with wound healing, which, along with systemic inflammatory response syndrome, is considered to be a normal post-operative condition. The pro-inflammatory microenvironment is also present during chemotherapy because of cellular senescence due to the acquired pro-inflammatory senescence-associated secretory phenotype of these cells. The same miRNAs have been indicated to be involved in wound healing, as similar mechanisms and pathways are responsible for inflammation. These miRNAs include the mentioned miR-21 and miR-155 (19), as well as miRNAs involved in angiogenesis, such as miR-16, miR-103, miR-191 and miR-21, the expression of which has been investigated in a study (20).

The aim of the present study was to evaluate a panel of seven circulating miRNAs in pre-operative, post-operative and 3-month follow-up samples to investigate changes in the level of selected miRNAs immediately after surgery and a longer time interval after the operation.

\section{Materials and methods}

Patients. Patients with diagnosed CRC or colorectal adenoma who underwent surgical intervention at the Clinic of Surgery and Transplant Centre, Jessenius Faculty of Medicine in Martin, Comenius University in Bratislava, (Martin, Slovakia) were enrolled in the present study. Patients were informed about the study and signed the informed consent form approved by the Ethical Committee at Jessenius Faculty in Martin, Comenius University in Bratislava (Martin, Slovakia) in accordance with the Declaration of Helsinki. The cohort included 110 patients with CRC or colorectal adenoma selected according to the following criteria: i) All patients were diagnosed with a defined clinical stage; ii) disease was confirmed by routine histopathological examination; and iii) none of the patients had any other disease or known malignancies that may have affected miRNA plasma levels.

Blood sample collection. Blood samples were collected between January 2018 and August 2020. For each patient, peripheral blood samples were collected two or three times and processed as soon as possible, no later than $1 \mathrm{~h}$ after collection. Pre-operative and post-operative blood samples were taken one day prior to surgery and 3-7 days after surgery, respectively. Another set of post-operative blood samples was collected during the follow-up period (3 months after surgical intervention). However, the follow-up group did not include blood samples from all patients $(n=20)$. Patients in the 
follow-up group did not receive any chemotherapy at the time of sampling.

Blood samples were collected into $\mathrm{K}_{3}$ EDTA tubes and transported $1 \mathrm{~h}$ after collection. The blood plasma was immediately separated from $10 \mathrm{ml}$ of whole blood by two-step centrifugation at $850 \mathrm{x} \mathrm{g}$ for $10 \mathrm{~min}$ at $4^{\circ} \mathrm{C}$ and $18,620 \mathrm{x} \mathrm{g}$ for $10 \mathrm{~min}$ at $4^{\circ} \mathrm{C}$ to completely remove components including cell debris, residual platelets and microvesicles. The plasma samples were stored at $-80^{\circ} \mathrm{C}$ until use.

Quality control of plasma samples, total RNA extraction and reverse transcription $(R T)$. The thawed plasma samples were visually inspected and the presence of free haemoglobin was measured by determining the absorbance at $414 \mathrm{~nm}$ by a Nanodrop ${ }^{\circledR} 1000$ (ThermoFisher Scientific, Inc.). Samples with an absorbance between 0.039 and 0.25 were included in the study. Only patients with pre-operative/post-operative $(n=60)$ and/or follow-up blood samples $(n=14)$ were included in the study. Total RNA including the miRNA fraction was extracted from $200 \mu \mathrm{l}$ of plasma using an miRNeasy Serum/Plasma Advanced kit (Qiagen $\mathrm{GmbH}$ ) following the manufacturer's protocol. RNA spike-in controls (RNA Spike-in kit; Qiagen $\mathrm{GmbH}$ ) were added into the lysis buffer to provide control of RNA extraction. Total RNA was resuspended in $20 \mu \mathrm{l}$ of RNase-free water and stored at $-80^{\circ} \mathrm{C}$ until RT. A total of $10 \mu \mathrm{l}$ of $\mathrm{RT}$ reaction mixture contained, in addition to common ingredients (miRCURY LNA RT kit; Qiagen GmbH), $1 \mu 1$ of total RNA and $0.5 \mu \mathrm{l}$ of RNA synthetic spike-ins. RT was performed in a thermal cycler for $1 \mathrm{~h}$ at $42^{\circ} \mathrm{C}$, followed by inactivation for $5 \mathrm{~min}$ at $95^{\circ} \mathrm{C}$. The obtained cDNA was stored at $-20^{\circ} \mathrm{C}$ until analysis, no longer than 5 weeks, and diluted at a $1: 30$ ratio prior to analysis.

Measurement of selected miRNAs by RT-quantitative (q) $P C R$. Based on a review of the literature (4-26), various circulating miRNAs (miR-106a-5p, miR-210-5p, miR-155-5p, miR-21-5p, miR-103a-3p, miR-191-5p, miR-16-5p) were selected and their levels were analysed. Quantification of each miRNA was performed in duplicate using an miRCURY LNA miRNA PCR System (Qiagen $\mathrm{GmbH}$ ). All pipetting steps were performed on a Bravo liquid handling station (Agilent Technologies, Inc.) and run in duplicate/triplicate on a LightCycler (LC)480 instrument (Roche Diagnostics $\mathrm{GmbH}$ ). Rapid quantification analysis was performed using LC480 instrument software and quantification cycle $(\mathrm{Cq})$ values were calculated by the second derivative method (27). Secondary analysis and quality control of RNA isolation and RT through synthetic spike-ins were performed using the GeneGlobe data analysis tool (Qiagen $\mathrm{GmbH}$ ). The web tool (https://geneglobe.qiagen.com/sk/analyze) was also used for rough data analysis, although no suitable reference genes were found with the geNorm or Normfinder algorithms, as all tested groups had different average arithmetic means.

Statistical analysis. The data were visualized and analysed using R version 3.5.2 (28). Expression and fold changes (FC) were computed using the standard formula (29) for a paired design. Furthermore, normalization of $\mathrm{Cq}$ values gene by gene $(\Delta \mathrm{Cq})$ and between groups is important to minimize the technical variability and the FC is the expression ratio of the
miRNA $(-\Delta \Delta \mathrm{Cq})$ between two conditions. The null hypothesis that the population median FC is equal to and/or is equal for two levels of a categorical clinical parameter (e.g., sex) was tested by the Wilcoxon rank-sum test. The analogous null hypothesis for a categorical clinical parameter with $>2$ levels (e.g., Cq) was tested by the Kruskal-Wallis test. The association between two clinical parameters (e.g., sex and Cq) was examined by the $\chi^{2}$ test. $\mathrm{P}<0.05$ was considered to indicate a statistically significant difference.

Analysis of involvement of differentially expressed miRNAs in signalling pathways associated with CRC relapse. The involvement of differentially expressed miRNAs (miR-106a-5p, miR-21-5p and miR-16-5p) in signalling pathways associated with CRC relapse was analysed through the online software DIANA mirPath v.3 (http://snf-515788. vm.okeanos.grnet.gr/) from the Kyoto Encyclopedia of Genes and Genomes (KEGG) based on analysis of functional pathway enrichment, as well as numerous segments of Gene Ontology (GO) analysis in the Homo sapiens species (30). The online analysis tool was used for combining the available in silico-predicted targets (DIANA-microT-CDS and/or TargetScan v6.2 algorithm) and high-quality experimentally supported interactions (DIANA-TarBase v7.0 algorithm), and $\mathrm{P}<0.05$ was considered to indicate statistical significance. In the KEGG analysis, gene intersections with all three miRNAs included were preferred and in the GO analysis, category intersections for merging the results with false discovery rate correction were chosen.

\section{Results}

Patient characteristics. Overall, 110 patients of Caucasian ethnicity with diagnosed CRC or adenoma were recruited for the present study. As indicated in Fig. 1, 30 patients were excluded due to other malignancies or inoperable tumours. The plasma samples of the remaining 80 patients were analysed. Extraction of RNA including the miRNA fraction was performed on 60 samples without haemolysis. If haemolysis was present in the first or second sample taken at different time points, the patient was excluded from analysis. It is known that the circulating miRNA profile may be significantly influenced by haemolysis of red blood cells, which does not mirror the physiological release from cells, including cancer cells (31).

The patient characteristics are presented in Table I. The patients' mean age at the time-point of diagnosis was 69 years [interquartile range (IQR), 63-73 years] for males $(n=41)$ and 68 years (IQR, 58-72 years) for females $(n=19)$. Carcinoma was present in almost $83 \%$ of males and $78 \%$ of females. Most patients were at stage II or III and in 54\% of males and $74 \%$ of females, tumours were localized in the proximal colon. Table II contains additional information on neoadjuvant treatment and follow-up. Disease recurrence was present in $40 \%$ of males and in $31 \%$ of females. Regrettably, it was not possible to acquire follow-up blood samples from $78.1 \%$ of males and $73.7 \%$ of females due to low compliance of the patients. Furthermore, the situation was complicated by the coronavirus pandemic. The information regarding patient relapse is incomplete, as patients were also managed at other hospitals and there is no national central register in Slovakia. 


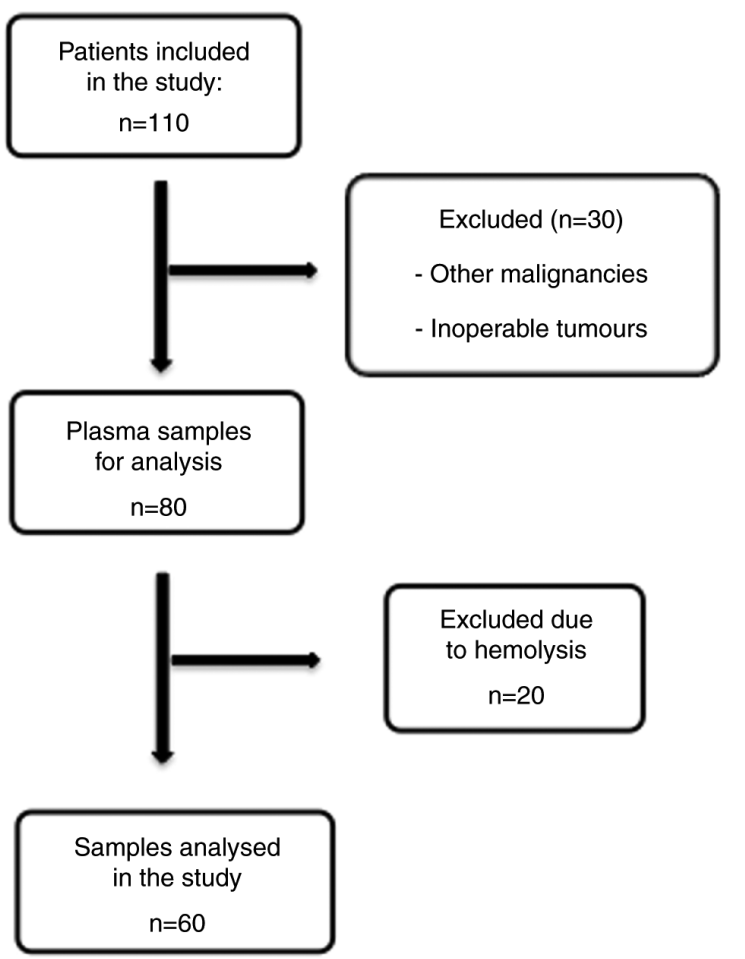

Figure 1. Schematic of patient and sample inclusion and/or exclusion in the study.

Evaluation of $C q$ values determined by miRNA expression analysis of plasma. Most of the selected miRNAs (miR-106a, $-21,-103 a,-191$ and -16 ) were detected in plasma samples with calculated $\mathrm{Cq}$ values between 22 and 35 using the second derivative method. A small portion of analysed miRNAs (miR-210-5p and miR-155-5p) had $\mathrm{Cq}>35$, which means weak plasma expression and these miRNAs were not included in the general conclusions of the study. Secondary analysis using the GeneGlobe data analysis tool revealed that RNA isolation and RT were performed correctly. During the data normalization process, differences in average arithmetic mean between tested groups from 0.5 to $1 \mathrm{Cq}$ were indicated. No combinations of selected miRNAs were appropriate for data normalization without calculation of the stability factor due to missing $\mathrm{Cq}$ values for certain samples. Normalization to the synthetic spike-in Caenorhabditis elegans miR-39 (cel-miR-39) was also not appropriate, as it is not affected by the biological condition of the patient and input concentration of miRNA. Therefore, in FC calculations, the median of all measured miRNAs except spike-ins was used, as recommended in the literature (32). In the statistical analysis of FC and the P-value calculation, post-operative and follow-up samples were compared to pre-operative samples.

Circulating miRNA expression in post-vs. pre-operative samples from patients with CRC. The results of the miRNA analysis of samples acquired pre- and post-operatively indicated significant upregulation of miR-21-5p $(\mathrm{P}=0.010)$, miR-155-5p $(\mathrm{P}=0.004)$ and miR-191-5p $(\mathrm{P}=0.005)$, and downregulation of miR-16-5p ( $\mathrm{P}=0.006)$ (Table III; Fig. 2).

Comparison of miRNA expression with histopathological parameters indicated significant upregulation of miR-210-5p in the circulation of patients who underwent neoadjuvant therapy
Table I. Characteristics of patients included in the study.

\begin{tabular}{|c|c|c|}
\hline Item & Males $(n=41)$ & Females $(n=19)$ \\
\hline Age, years & $69(63 ; 73)$ & $68(58 ; 72)$ \\
\hline \multicolumn{3}{|l|}{ Tumor type } \\
\hline Carcinoma & $34(82.9)$ & $15(78.0)$ \\
\hline Adenoma & $2(4.9)$ & $2(11.0)$ \\
\hline WNL & $5(12.2)$ & $2(11.0)$ \\
\hline \multicolumn{3}{|l|}{ Tumor localization } \\
\hline Rectum, distal colon & $19(46.0)$ & $5(26.0)$ \\
\hline Proximal colon & $22(54.0)$ & $14(74.0)$ \\
\hline \multicolumn{3}{|l|}{ Stage } \\
\hline I & $7(17.1)$ & $2(10.5)$ \\
\hline II & $10(24.4)$ & $3(15.8)$ \\
\hline III & $10(24.4)$ & $9(47.4)$ \\
\hline IV & $4(9.7)$ & $2(10.5)$ \\
\hline Adenomas/polyps & $10(24.4)$ & $3(15.8)$ \\
\hline \multicolumn{3}{|l|}{ pT } \\
\hline 0 & $1(2.4)$ & $0(0.0)$ \\
\hline 1 & $6(14.6)$ & $0(0.0)$ \\
\hline 2 & $6(14.6)$ & $2(10.5)$ \\
\hline 3 & $15(36.6)$ & $8(42.1)$ \\
\hline 4 & $7(17.2)$ & $4(21.1)$ \\
\hline Adenomas/polyps & $6(14.6)$ & $5(26.3)$ \\
\hline \multicolumn{3}{|l|}{$\mathrm{pN}$} \\
\hline 0 & $22(53.7)$ & $7(36.8)$ \\
\hline 1 & $8(19.5)$ & $3(15.8)$ \\
\hline 2 & $9(21.9)$ & $4(21.1)$ \\
\hline Unknown & $2(4.9)$ & $5(26.3)$ \\
\hline \multicolumn{3}{|l|}{$\mathrm{pM}$} \\
\hline 0 & $2(4.9)$ & $2(10.5)$ \\
\hline 1 & $4(9.8)$ & $3(15.8)$ \\
\hline $\mathrm{X}$ & $35(85.3)$ & $11(57.9)$ \\
\hline Adenomas/polyps & $0(0.0)$ & $3(15.8)$ \\
\hline \multicolumn{3}{|l|}{ Grade } \\
\hline 1 & $14(34.1)$ & $2(10.5)$ \\
\hline 2 & $12(29.3)$ & $7(36.8)$ \\
\hline 3 & $4(9.8)$ & $3(15.8)$ \\
\hline Adenomas/polyps & $11(26.8)$ & $7(36.8)$ \\
\hline \multicolumn{3}{|l|}{ Microsatellite stability } \\
\hline MSS & $32(78.1)$ & $8(42.1)$ \\
\hline MSI & $1(2.4)$ & $5(26.3)$ \\
\hline Adenomas/polyps & $8(19.5)$ & $6(31.6)$ \\
\hline \multicolumn{3}{|l|}{ Relapse } \\
\hline Absent & $15(36.6)$ & $9(47.4)$ \\
\hline Present & $10(24.4)$ & $4(21.1)$ \\
\hline Unknown & $16(39.0)$ & $6(31.5)$ \\
\hline
\end{tabular}

Values are expressed as $\mathrm{n}(\%)$, except for age, which is expressed as the median (lower quartile; upper quartile). WNL, without neoplastic lesion-patients after neoadjuvant treatment; MSS, microsatellite stable; MSI, microsatellite instable.

$(\mathrm{P}=0.012)$. When excluding patients with neoadjuvant therapy from the data, miR-106a-5p expression was significantly 
Table II. Distribution of male and female patients according to neoadjuvant treatment and 3 months of follow-up.

\begin{tabular}{lcc}
\hline Item & Males, n (\%) & Females, n (\%) \\
\hline $\begin{array}{l}\text { Neoadjuvant therapy } \\
\text { Yes }\end{array}$ & $5(12.2)$ & $1(5.3)$ \\
No & $36(87.8)$ & $18(94.7)$ \\
Follow-up after 3 m & & \\
Carcinoma & $9(21.9)$ & $4(21.0)$ \\
Adenoma & $0(0.0)$ & $1(5.3)$ \\
No 3 m follow-up & $32(78.1)$ & $14(73.7)$ \\
Relapse & & \\
Absent & $15(36.6)$ & $9(47.4)$ \\
Present & $10(24.4)$ & $4(21.1)$ \\
No information available & $16(39.0)$ & $6(31.5)$ \\
\hline
\end{tabular}

Values are expressed as n (\%). 3 m, 3 months.

increased with rising severity of the stage $(\mathrm{P}=0.033)$. No association with tumour type, stage, size, nodal status, metastases, grade or relapse was obtained. No significant associations were observed between miR-21-5p, miR-103a-3p, miR-155-5p, miR-191-5p and miR-16-5p expression and histopathological parameters. All P-values are listed in Table SI.

Circulating miRNA expression in plasma of patients with CRC in follow-up samples compared to pre-operative levels. The expression of selected miRNAs in follow-up samples $(n=14)$ was compared to that in pre-operative plasma samples $(n=60)$. The results suggested that miR-106a-5p, miR-191-5p and $\mathrm{miR}-16-5 \mathrm{p}$ were significantly upregulated $(\mathrm{P}=0.005,0.048$ and 0.003 , respectively) in follow-up samples taken 3 months after surgery when compared to the condition before surgery (Fig. 3). However, miR-210-5p ( $\mathrm{P}=0.1309)$, miR-155-5p $(\mathrm{P}=0.1099)$, miR-21-5p $(\mathrm{P}=0.1353)$ and miR-103a-3p $(\mathrm{P}=0.052)$ did not exhibit any statistically significant changes (Table III).

Statistical analyses were performed to assess any possible associations between miRNA expression and pathological parameters. However, the expression of these seven miRNAs was not statistically significantly associated with the TNM stage, stage, grade or tumour type of the patients (data not shown).

Circulating miRNA expression in paired post-operative and follow-up samples. Changes in the expression levels of the selected panel of miRNAs in paired post-operative and follow-up samples compared to the pre-operative levels are graphically presented in Fig. 4. Analysis of outliers for patients with known histopathological data for each of the selected miRNAs identified follow-up samples from two patients with metastasis in the liver at the time of follow-up sampling with a different profile of miR-210 and miR-21 expression

A summary of the median FCs in paired post-operative and follow-up samples for each investigated miRNA is presented in Table IV. Circulating miR-106a-5p and miR-16-5p were significantly upregulated $(\mathrm{P}=0.027$ and 0.003 , respectively) in the follow-up samples and miR-21-5p was significantly downregulated $(\mathrm{P}=0.035)$.

Signal and functional pathway analysis. The target genes and function of the most differentially expressed miRNAs associated with tumour removal, wound healing and possible recurrence were analysed by KEGG pathway analysis using the DIANA-mirPath v3.0 online web analysis tool. miR-106a-5p was experimentally proven to have 1,160 target genes, miR-16-5p has 2,886 target genes and miR-21-5p has 1,372 target genes listed in DIANA-TarBase algorithm (accessed, 30 November 2020). In a gene intersection setting for merging the results of the KEGG analysis (Table V), it was indicated that all three miRNAs are significantly enriched in pathways involving proteoglycans in cancer (hsa05205; 9 target genes), the Hippo signalling pathway (hsa04390; 5 target genes), focal adhesion (hsa04510; 11 target genes), signalling pathways regulating pluripotency of stem cells (hsa04550; 7 target genes), the prolactin signalling pathway (hsa04917; 5 target genes), endocrine and other factor-regulated calcium reabsorption (hsa04961; 2 target genes), pathways in cancer (hsa05200: 8 target genes), CRC (hsa05210; 4 target genes), the FoxO signalling pathway (hsa04068; 8 target genes), endometrial cancer (hsa05213; 3 target genes), thyroid cancer (hsa05216; 3 target genes), lysine degradation (hsa00310; 2 target genes) and the p53 signalling pathway (hsa04115; 5 target genes). Three putative target genes (CCND1, CTNNB1 and MAPK1) were indicated to be associated with 6 of the 11 signalling pathways (proteoglycans in cancer, focal adhesion and pathways in cancer, as well as colorectal, endometrial and thyroid cancer).

Investigation of the biological function of target genes regulated by differentially expressed miRNAs in the subcategories biological process, cellular component or molecular function, was performed through GO analysis. GO annotation results for category intersection enrichment of target genes influenced by up- or downregulated miRNAs in patients' follow-up samples are provided in Table VI. The most enriched GO annotations were 'mitotic cell cycle' (GO:0000278), 'otein complex assembly' (GO:0006461) and 'cellular protein modification process' (GO:0006464). The top $10 \mathrm{GO}$ terms in the biological process category were presented and a full list of GO terms is provided in Table SII. A visualization of GO terms in each category is displayed in Fig. 5.

\section{Discussion}

Numerous studies have focused on miRNAs and their prognostic and predictive roles in CRC. Various studies had conflicting results regarding the up- and downregulation of selected miRNAs in assessing the prognosis and therapy response. In the present study, a group of seven miRNAs were selected from the literature that were significantly upor downregulated post-operatively and in follow-up samples when compared to the pre-operative state.

Within the selected panel of circulating miRNAs, the plasma expression of miR-106a-5p was not significantly changed in post-operative samples. However, miR-106a-5p expression was significantly increased in follow-up samples $(\mathrm{P}=0.005)$. By contrast, certain studies have demonstrated 
Table III. Fold-changes in the levels of selected miRNAs in the post-operative and follow-up samples collected 3 months after surgery compared with pre-operative stages.

\begin{tabular}{lcccr}
\hline miRNA & Post-operative samples $(\mathrm{n}=60)$ & P-value & Follow-up samples $(\mathrm{n}=14)$ & P-value \\
\hline miR-106a-5p & $1.04(0.91,1.18)$ & 0.513 & $3.48(1.54,5.88)$ & 0.005 \\
miR-210-5p & $1.47(0.94,2.29)$ & 0.086 & $3.15(0.8,12.03)$ & 0.130 \\
miR-155-5p & $1.9(1.26,3.67)$ & 0.004 & $3.46(0.88,8.24)$ & 0.110 \\
miR-21-5p & $1.15(1.04,1.3)$ & 0.010 & $0.65(0.46,1.43)$ & 0.140 \\
miR-103a-3p & $1.01(0.83,1.26)$ & 0.900 & $0.69(0.54,1.04)$ & 0.052 \\
miR-191-5p & $1.2(1.06,1.37)$ & 0.005 & $1.48(1.01,2.17)$ & 0.048 \\
miR-16-5p & $0.83(0.71,0.94)$ & 0.006 & $1.73(1.16,2.65)$ & 0.003 \\
\hline
\end{tabular}

Values are expressed as the median (interquartile range). Wilcoxon's rank-sum test was performed for statistical comparisons. miRNA/miR, microRNA.
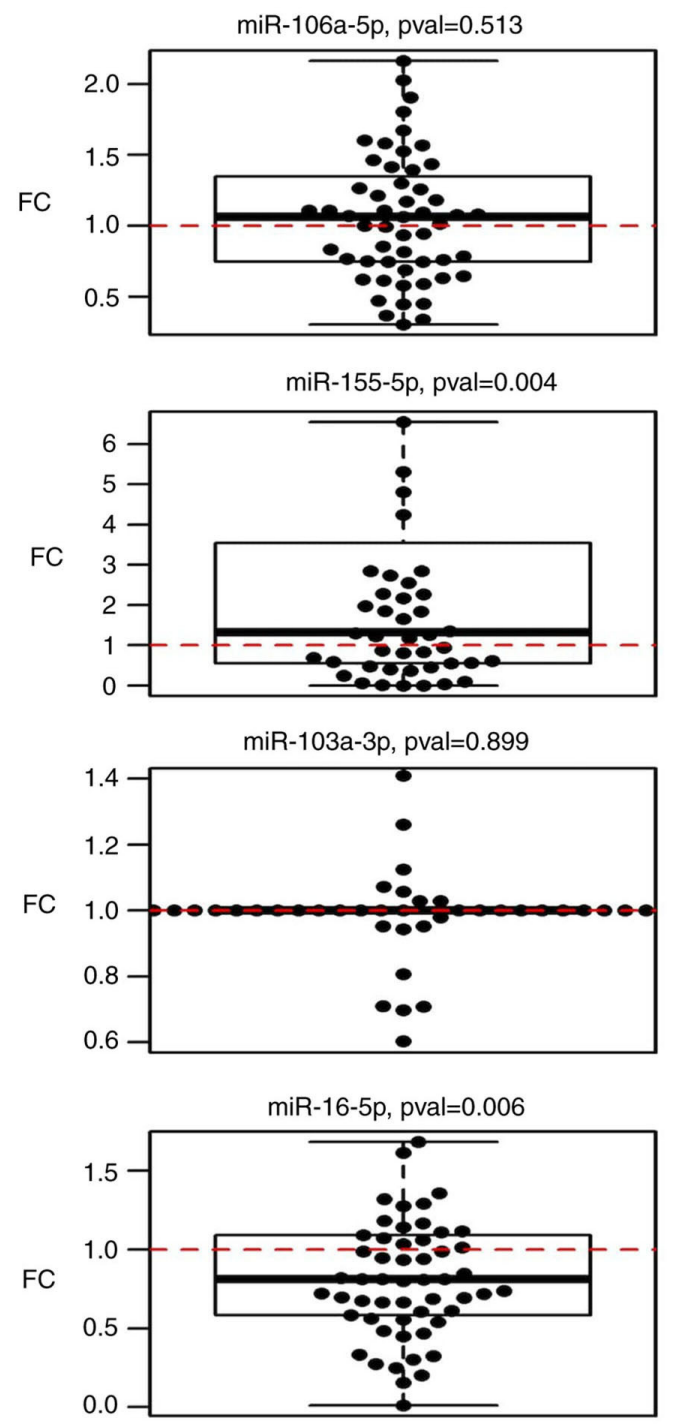
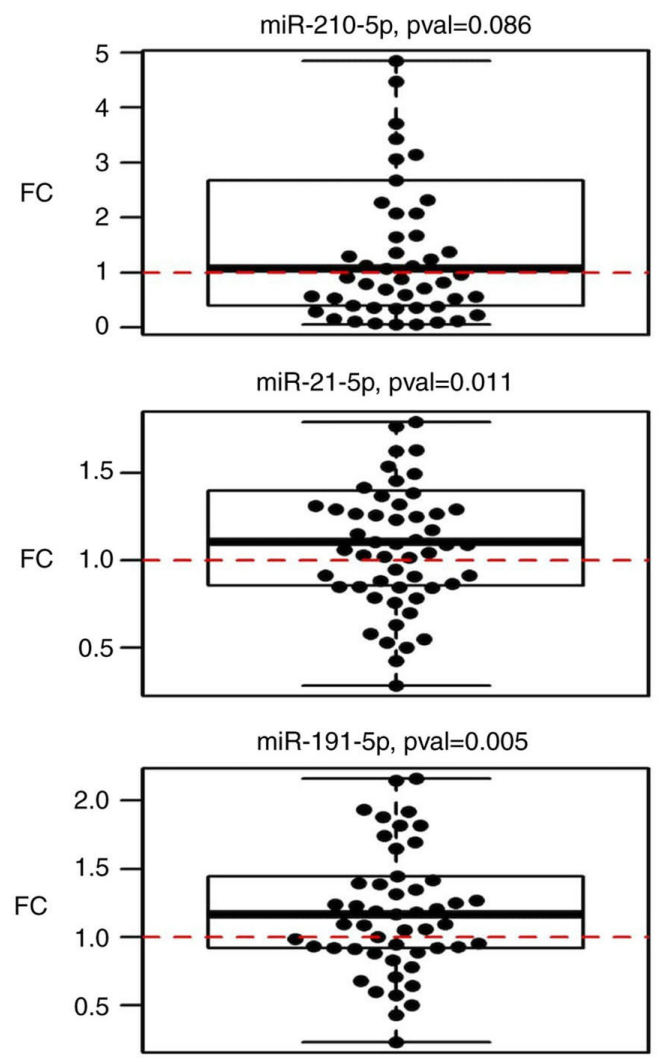

Figure 2. Boxplots displaying circulating miRNA expression in post-operative samples compared to pre-operative expression. FC, fold change; miR, microRNA; pval, P-value.

higher plasma $(6,33)$ and/or serum (34) miR-106a expression in patients with CRC than in healthy individuals and lower miR-106 levels in post-operative samples (35). Li et al (34) also indicated that high miR-106a expression in patients with CRC treated with adjuvant chemotherapy is associated with poor therapeutic outcome and shorter disease-free survival (DFS). 


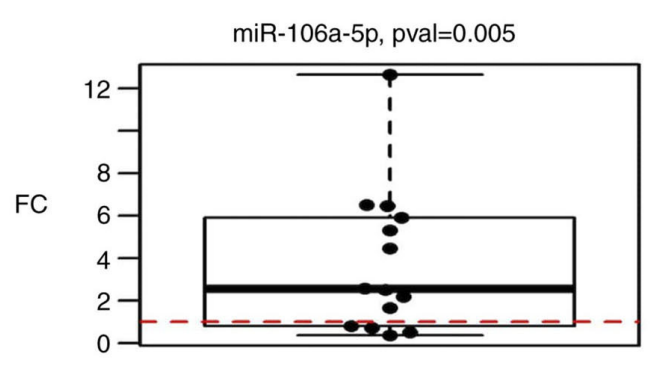

miR-155-5p, pval=0.11

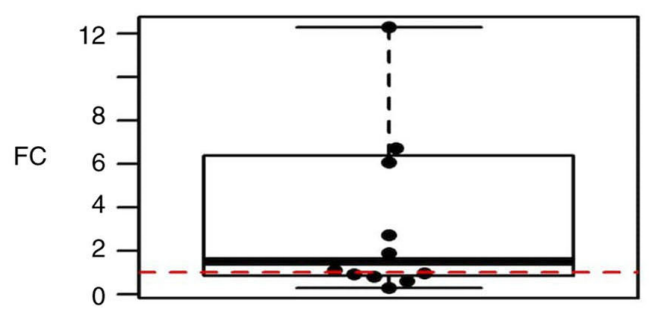

miR-103a-3p, pval=0.052

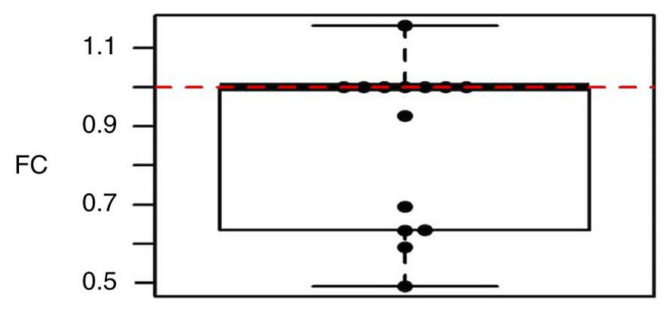

miR-16-5p, pval=0.003

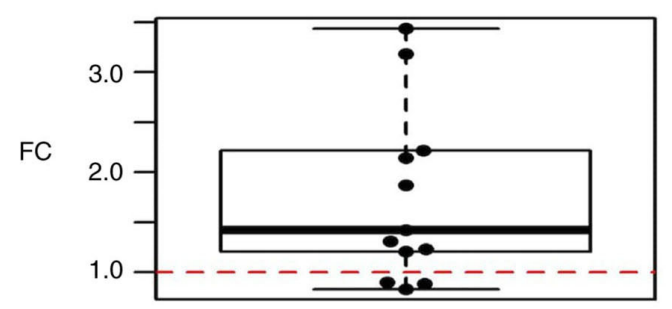

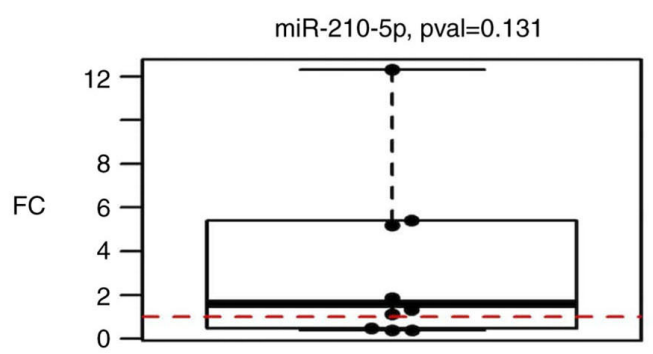

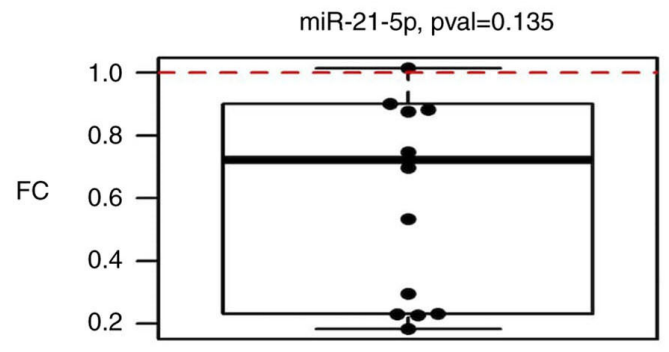

miR-191-5p, pval=0.048

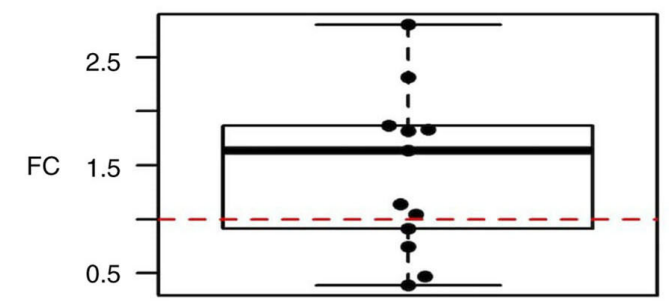

Figure 3. Boxplots displaying the expression of selected circulating miRNAs in follow-up samples ( 3 months after surgery) compared to the pre-operative state. FC, fold change; miR, microRNA; pval, P-value.

In addition, increased levels of miR-106a have been detected in CRC tissue compared to normal adjacent tissue, and downregulation of miR-106a in CRC tissue has been reported as a negative prognostic marker in patients with CRC (36). On the other hand, in one study, miR-106a levels in plasma of patients with CRC exhibited no significant difference in the early post-operative period and increased 1 month after surgery (37). The results of the present study are in line with these results, although the pre-operative expression was not compared with a healthy cohort.

Studies have suggested that miR-106a is involved in the MAPK signalling pathway, focal adhesion, the FoxO signalling pathway, CRC and other malignancies (38). miR-106a has been demonstrated to be upregulated in metastatic CRC tissue and to be associated with advanced TNM stage and lymph node metastasis. Furthermore, miR-106a directly targets DLC1 and thus promotes $\mathrm{CRC}$ cell migration and invasion through deregulation of the Wnt/ $\beta$-catenin signalling pathway (39). Upregulation of miR-106a-5p in plasma of follow-up samples may be associated with cell migration and proliferation during intestinal healing that is accompanied by similar signalling pathways as cancer.

As mentioned in the Results, the expression level of miR-155 and miR-210 in the present study was difficult to evaluate due to higher $\mathrm{Cq}$ values $(>35 \mathrm{Cq}$ ) and a high standard deviation from triplicate experiments. A slightly higher expression level was detected in post-operative samples and no change in follow-up samples. High expression of miR-155 and miR-210 has been reported in CRC tissue compared to normal adjacent tissue (40-42). The serum levels of miR-155 and -210 decreased significantly 3 months after surgery and chemotherapy and were elevated again at 12-18 months after treatment and prior to the diagnosis of distant metastasis and recurrence $(5,26)$.

Sabry et al (43) found that upregulation of miR-210 is associated with large tumour size, positive lymph node metastasis and local invasion. Qu et al (42) also suggested that upregulation of miR-210 is induced by hypoxia, which was confirmed by another study (44). Concerning the expression of miR-210, the present results did not reveal any statistically 

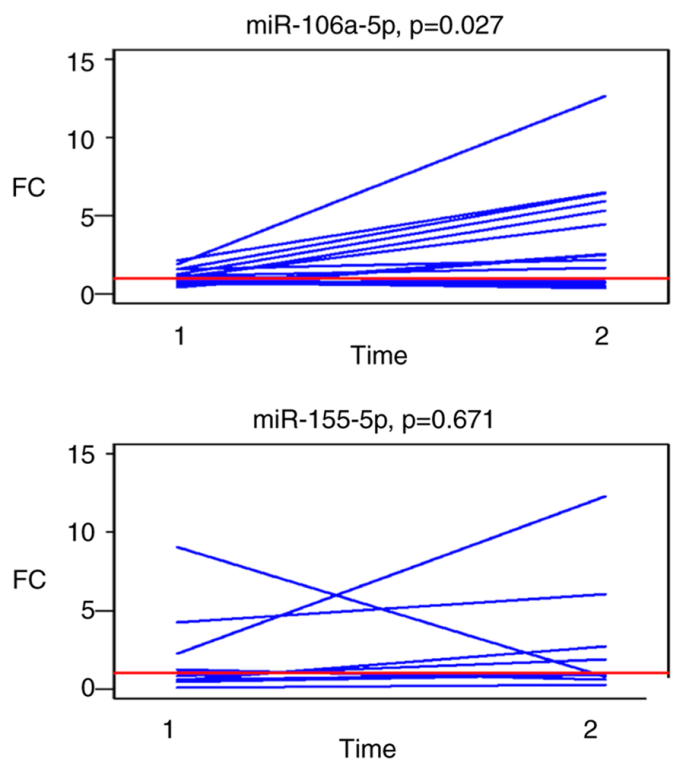

miR-103a-3p, $p=0.093$
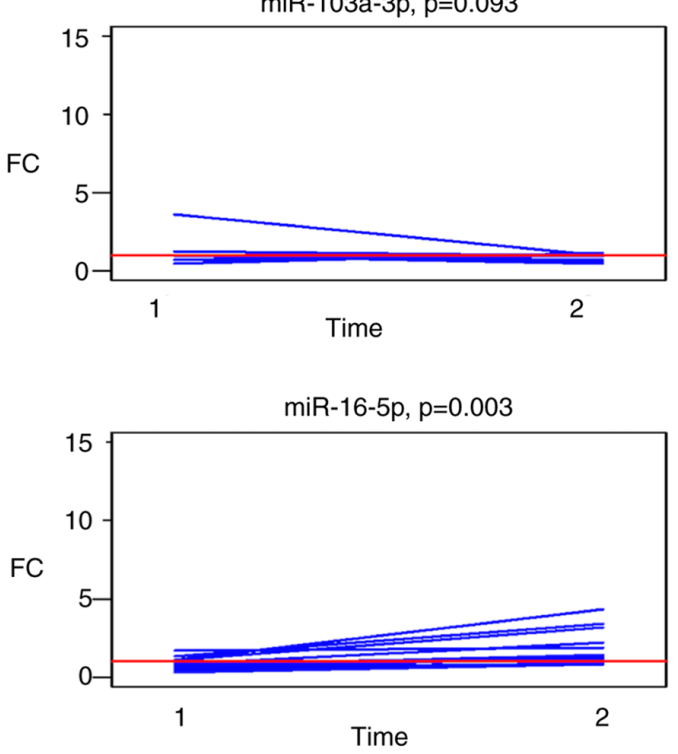
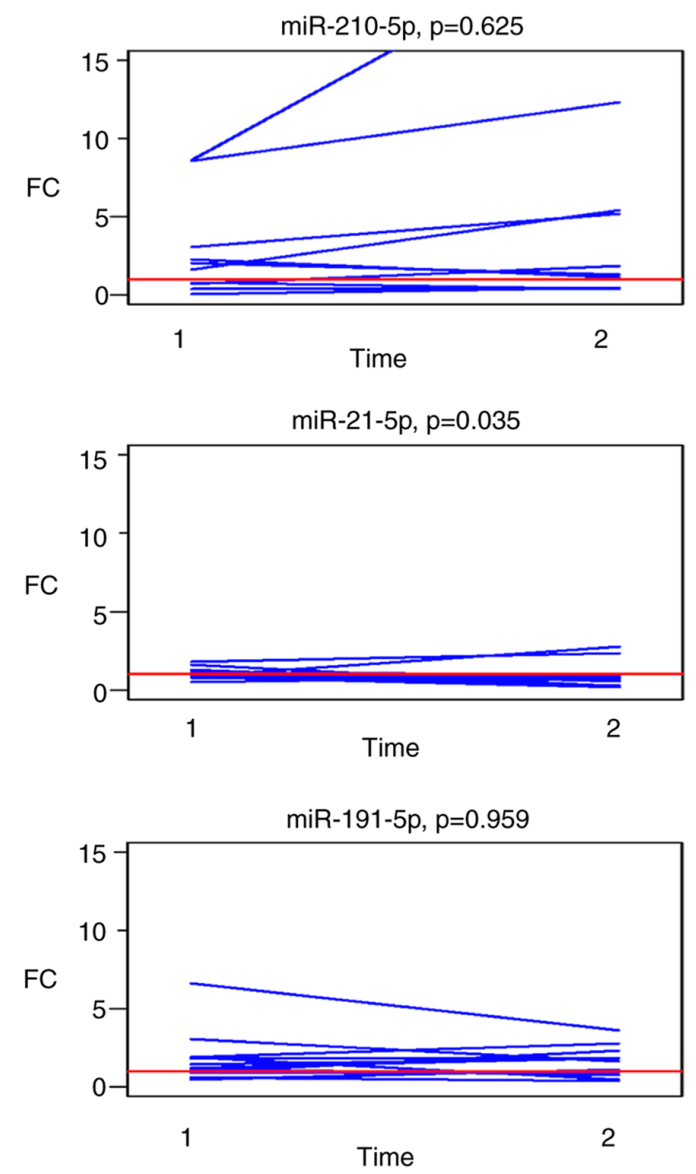

Figure 4. FCs in the circulating miRNA expression levels in paired samples from 14 patients: Post-operative samples (time-point 1) and in follow-up samples collected 3 months after surgery (time-point 2; indicated on the X-axis). Paired values are connected by lines. FC, fold change; miR, microRNA.

significant changes; however, Sabry et al (43) determined higher expression of miR-210 in patients with CRC and adenomas. Furthermore, the present study indicated higher miR-210 expression in patients who underwent neoadjuvant treatment. Jung et al (45) reported that patients with breast cancer treated with neoadjuvant chemotherapy had elevated circulating miR-210-5p and suggested that miR-210 may be used to predict the outcome and monitor therapeutic response.

miR-155 has an important role in the immune system due to targeting $\sim 140$ genes encoding immunomodulatory proteins, inflammation-related proteins and tumour suppressor proteins (46). miR-155 has been indicated to be upregulated in numerous malignancies as well as CRC (47) where chronic inflammation has an important role (48). Furthermore, miR-155 downregulates core MMR proteins and induces microsatellite instability (MSI) in CRC. Therefore, upregulation of miR-155 may have a role in tumorigenesis by combining MSI and inflammatory stimuli (49). Ulivi et al (50) analysed a panel of circulating miRNAs in relation to the outcome in patients with metastatic CRC treated with bevacizumab. They determined that miR-155 upregulation one month after bevacizumab treatment was associated with significantly shorter progression-free survival and overall survival (OS), and thus, miR-155 may be utilized in drug response monitoring (50).

In the present study, significant upregulation of miR-21 in post-operative samples compared to the pre-operative state and significant downregulation in follow-up samples compared to paired post-operative samples was observed. Jin et al (8) reported upregulation of miR-21 in pre-operative samples, post-operative miR-21 downregulation and no difference in patients with recurrence. miR-21 is an important molecule in epithelial-mesenchymal transition (EMT) and its low expression is associated with increased OS. Equivalently, higher serum miR-21 expression in patients with CRC was reported to be associated with liver metastasis and CRC recurrence (12), as well as poorer DFS and OS (24). miR-21 
Table IV. Differences in the post- and follow-up vs. pre-operative levels of miRNAs from the selected panel (fold changes).

\begin{tabular}{lccr}
\hline miRNA & Post-operative samples $(\mathrm{n}=14)$ & Follow-up samples $(\mathrm{n}=14)$ & P-value \\
\hline miR-106a-5p & $1.09(0.78,1.50)$ & $2.54(1.01,5.77)$ & 0.027 \\
miR-210-5p & $1.5(0.7,2.3)$ & $1.6(0.6,5.4)$ & 0.600 \\
Unknown & 0 & 4 & 0.700 \\
miR-155-5p & $1.2(0.6,3.1)$ & $1.5(0.9,6.2)$ & \\
Unknown & 2 & $0.72(0.25,0.90)$ & 0.035 \\
miR-21-5p & $1.02(0.85,1.22)$ & $1.00(0.65,1.00)$ & 0.093 \\
miR-103a-3p & $1.00(1.00,1.00)$ & $1.64(0.91,1.87)$ & $>0.900$ \\
miR-191-5p & $1.21(0.93,1.92)$ & 1 & 0.003 \\
Unknown & 1 & $1.42(1.21,2.22)$ & \\
miR-16-5p & $0.81(0.63,1.10)$ & 1 & \\
Unknown & 2 &
\end{tabular}

Values are expressed as the median (lower quartile, upper quartile). Wilcoxon's rank-sum test was performed for statistical comparisons. miRNA/miR, microRNA.

Table V. KEGG analysis of the significantly deregulated microRNAs in patients' plasma three months after surgery with related target genes.

\begin{tabular}{|c|c|c|c|}
\hline KEGG pathway & Pathway ID & P-value & Targeted genes \\
\hline Proteoglycans in cancer & hsa05205 & $6.6041 \times 10^{-7}$ & $\begin{array}{l}\text { STAT3, PDCD4, FRS2, IGF1R, CCND1, CTNNB1, } \\
\text { TIMP3, VEGFA, MAPK1 }\end{array}$ \\
\hline Hippo signaling pathway & hsa04390 & $8.3834 \times 10^{-5}$ & YAP1, CCND2, CCND1, CTNNB1, LATS1 \\
\hline Focal adhesion & hsa04510 & 0.0076 & $\begin{array}{l}\text { ITGB8, PAK2, CCND2, IGF1R, ARHGAP35, CCND1, } \\
\text { CTNNB1, VEGFA, MAPK1, TLN1, COL4A1 }\end{array}$ \\
\hline $\begin{array}{l}\text { Signaling pathways regulating } \\
\text { pluripotency of stem cells }\end{array}$ & hsa04550 & 0.0096 & STAT3, REST, IGF1R, ZFHX3, CTNNB1, SKIL, MAPK1 \\
\hline Prolactin signaling pathway & hsa04917 & 0.0096 & STAT3, CCND2, SOCS6, CCND1, MAPK1 \\
\hline $\begin{array}{l}\text { Endocrine and other factor-regulated } \\
\text { calcium reabsorption }\end{array}$ & hsa04961 & 0.0096 & CLTC, RAB11A \\
\hline Pathways in cancer & hsa05200 & 0.0096 & $\begin{array}{l}\text { STAT3, IGF1R, APPL1, CCND1, CTNNB1, VEGFA, } \\
\text { MAPK1, COL4A1 }\end{array}$ \\
\hline Colorectal cancer & hsa05210 & 0.0096 & APPL1, CCND1, CTNNB1, MAPK1 \\
\hline FoxO signaling pathway & hsa04068 & 0.0167 & $\begin{array}{l}\text { STAT3, CCND2, IGF1R, CCND1, PRKAB2, SOD2, } \\
\text { MAPK1, CCNG2 }\end{array}$ \\
\hline Endometrial cancer & hsa05213 & 0.0167 & CCND1, CTNNB1, MAPK1 \\
\hline Thyroid cancer & hsa05216 & 0.027 & CCND1, CTNNB1, MAPK1 \\
\hline Lysine degradation & hsa00310 & 0.0424 & WHSC1, KMT2C \\
\hline p53 signaling pathway & hsa04115 & 0.0424 & CCND2, CCND1, SESN1, TNFRSF10B, CCNG2 \\
\hline
\end{tabular}

KEGG, Kyoto Encyclopedia of Genes and Genomes; hsa, Homo sapiens.

expression in CRC tumour tissue was indicated to be elevated compared with that in normal adjacent tissue and miR-21 induces proliferation in CRC cell lines (51). Upregulation of miR-21 was also reported to be associated with a higher TNM stage and BRAF mutation (52). miR-21 promotes carcinogenesis by inhibiting negative regulation of the RAS/MEK/ERK pathway and also downregulates the expression of PTEN, TPM1 and PDCD4, promoting tumour progression (53). The present study indicated a slightly increased level of miR-21-5p expression immediately after surgery but a significant reduction in paired samples taken 3 months after surgery. In a study with a similar in patients with oesophageal squamous cell carcinoma, plasma expression of miR-21 was significantly reduced in post-operative samples taken 1 month after oesophagectomy (54). Furthermore, upregulation of miR-21 in post-operative samples may reflect an increased inflammatory response and oxidative stress with an increase in the oxidative stress response protein aldose reductase (AR) and 
Table VI. GO annotation results of the target genes of deregulated microRNAs in subcategories biological process (top 10 GO terms were included), cellular component and molecular function.

A, Biological process

\begin{tabular}{lllr}
\hline GO ID & \multicolumn{1}{c}{ GO term name } & P-value & Target gene count \\
\hline GO:0000278 & Mitotic cell cycle & $<1 \times 10^{-325}$ & 146 \\
GO:0006461 & Protein complex assembly & $<1 \times 10^{-325}$ & 239 \\
GO:0006464 & Cellular protein modification process & $<1 \times 10^{-325}$ & 744 \\
GO:0006950 & Response to stress & $<1 \times 10^{-325}$ & 625 \\
GO:0007596 & Blood coagulation & $<1 \times 10^{-325}$ & 150 \\
GO:0008150 & Biological_process & $<1 \times 10^{-325}$ & 3807 \\
GO:0008219 & Cell death & $<1 \times 10^{-325}$ & 305 \\
GO:0009056 & Catabolic process & $<1 \times 10^{-325}$ & 605 \\
GO:0009058 & Biosynthetic process & $<1 \times 10^{-325}$ & 1079 \\
GO:0010467 & Gene expression & $<1 \times 10^{-325}$ & 286 \\
\hline
\end{tabular}

B, Cellular component

\begin{tabular}{llrr}
\hline GO ID & \multicolumn{1}{c}{ GO term name } & P-value & Target gene count \\
\hline GO:0005575 & Cellular_component & $<1 \times 10^{-325}$ & 3927 \\
GO:0005654 & Nucleoplasm & $<1 \times 10^{-325}$ & 422 \\
GO:0005829 & Cytosol & $<1 \times 10^{-325}$ & 880 \\
GO:0043226 & Organelle & $<1 \times 10^{-325}$ & 2831 \\
GO:0043234 & Protein complex & $<1 \times 10^{-325}$ & 1092 \\
GO:0005815 & Microtubule organizing center & $3.363976 \times 10^{-14}$ & 140 \\
\hline
\end{tabular}

C, Molecular function

\begin{tabular}{llrr}
\hline GO ID & \multicolumn{1}{c}{ GO term name } & P-value & Target gene count \\
\hline GO:0000988 & Protein binding transcription factor activity & $<1 \times 10^{-325}$ & 165 \\
GO:0001071 & Nucleic acid binding transcription factor activity & $<1 \times 10^{-325}$ & 240 \\
GO:0003674 & Molecular_function & $<1 \times 10^{-325}$ & 3916 \\
GO:0003723 & RNA binding & $<1 \times 10^{-325}$ & 641 \\
GO:0008092 & Cytoskeletal protein binding & $<1 \times 10^{-325}$ & 244 \\
GO:0019899 & Enzyme binding & $<1 \times 10^{-325}$ & 475 \\
GO:0030234 & Enzyme regulator activity & $<1 \times 10^{-325}$ & 242 \\
GO:0043167 & Ion binding & $<1 \times 10^{-325}$ & 1563 \\
GO:0044822 & Poly(A) RNA binding & $<1 \times 10^{-325}$ & 532 \\
GO:0032182 & Small conjugating protein binding & $1.622062 \times 10^{-9}$ & 40
\end{tabular}

GO, Gene Ontology.

activation of PTEN-induced apoptosis and controlled cell growth. Repression of AR led to inhibition of colon cancer cell growth by downregulation of miR-21 expression and upregulation of PTEN and FOXO3a expression (hsa04068). However, the specific mechanism was not elucidated (55).

Hong et al (56) detected upregulation of miR-103 in CRC cell lines. Zheng et al (57) determined upregulation of miR-103 in CRC tissue compared to normal adjacent tissue. Furthermore, they demonstrated that patients with high miR-103 expression had poorer OS. Wang et al (58) reported upregulation of $\mathrm{miR}-103 \mathrm{a}-3 \mathrm{p}$ in serum of patients with CRC and in those with recurrence. There is currently a lack of studies evaluating the role of miR-103a-3p in carcinogenesis due to its use as a housekeeping gene as discussed below.

Another significantly upregulated miRNA in post-operative samples of the present study was miR-191-5p. There was also a weak significant association between the level of miR-191 and tumour stage and microsatellite-stable cancers. The function of miR-191 in CRC remains to be fully elucidated. However, 
A

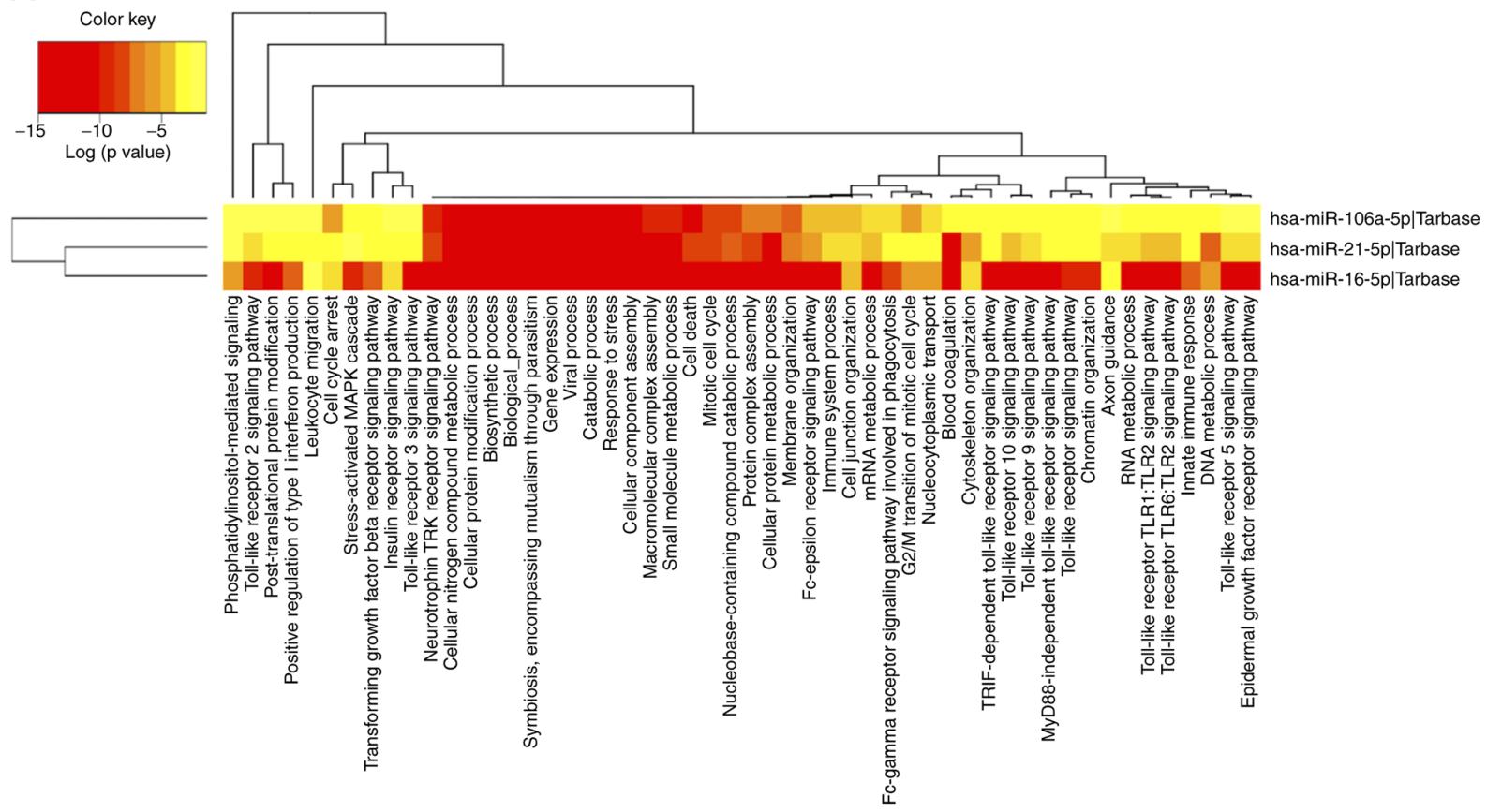

B

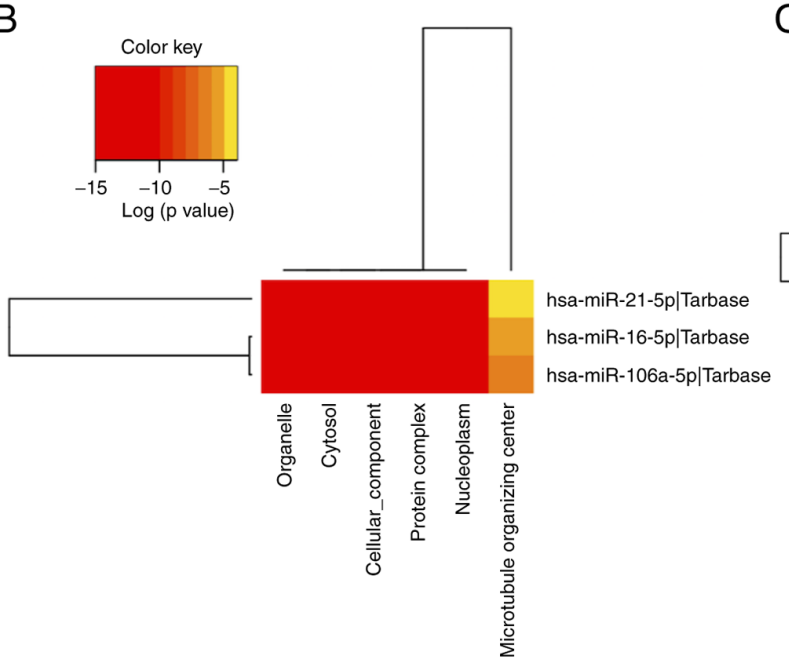

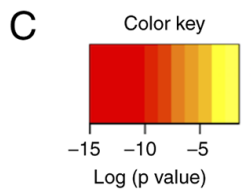

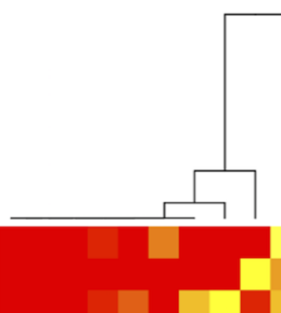

hsa-miR-21-5p|Tarbase hsa-miR-16-5p|Tarbase hsa-miR-106a-5p|Tarbase

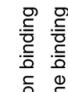

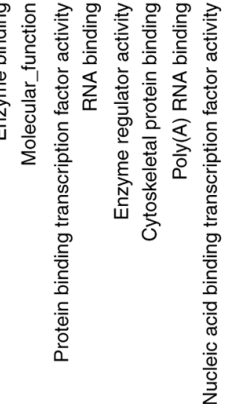

Figure 5. Heatmaps of GO analysis. GO enrichment of supposed targets was performed for three significantly deregulated miRNAs at three subcategories: (A) Biological process, (B) cellular component and (C) molecular function. Diverse levels of enrichment of pathway terms in the miRNA target genes are displayed by different colors, the red represents a higher degree of enrichment. GO, Gene Ontology; FC, fold change; miR, microRNA, hsa, Homo sapiens.

several studies have reported deregulation of miR-191 in various cancer types and diseases. It has been indicated that miR-191 overexpression induces progression of hepatocellular carcinoma and intrahepatic cholangiocarcinoma and promotes EMT in metastatic bronchial epithelial cells (59). Zhang et al (60) reported that upregulation of miR-191 promoted proliferation in CRC and reduced cell susceptibility to 5-fluorouracil. Qin et al (61) demonstrated that high expression of miR-191 is significantly associated with advanced TNM stage, liver metastasis and unfavorable prognosis in patients with CRC. On the other hand, Milanesi et al (62) and Chen et al (63) revealed that downregulation of miR-191 in patients with KRAS-mutation CRC and low miR-191 expression is correlated with poor prognosis.
In the present study, the level of miR-16-5p expression in patients' plasma after surgery was significantly decreased $(\mathrm{P}=0.006)$ compared to the pre-operative state and elevated in paired follow-up samples $(\mathrm{P}=0.003)$. Previous studies have demonstrated a tumour-suppressive role of miR-16 in the progression of several malignancies (64), including CRC (65). It has been indicated that overexpression of miR-16 inhibited proliferation and induced apoptosis by regulation of the p53/survivin signalling pathway (66). Several studies have revealed downregulation of miR-16 in malignant CRC tissue when compared to adjacent tissue and identified an association between low miR-16 expression and histological parameters such as advanced TNM stage and poor histological grade, as well as a higher incidence of lymph node metastases and 
tumour recurrence. Low miR-16 expression is correlated with shorter DFS and OS $(64,65,67)$. Ostenfeld et al (68) analysed miRNA profiles in epithelial-derived extracellular vesicles (EVs) secreted by cancer cells and observed a significantly reduced level of miR-16-5p post-operatively.

miR-16-5p, miR-103a-3p and miR-191-5p are commonly used for qPCR data normalization, which is a critical step in each gene expression experiment. Danese et al (25) aimed to identify the ideal reference miRNAs in CRC from miRTarBase and came to the conclusion that miR-16 and -103 target onco/tumour suppressor genes are not suitable for normalization. The expression of miR-16-5p in blood plasma is influenced by a varying level of haemolysis (29). Therefore, in the present study, samples without haemolysis were carefully selected despite the overall reduction in the number of samples in the test groups. Proper data normalization is the most important part of each study and may influence its overall results. In studies dealing with circulating miRNA expression, the issue prevails that there are no suitable housekeeping miRNAs expressed equally across the various physiological conditions of patients. Other procedures advise normalization to synthetic spike-ins added through the process of sample preparation prior to analysis. However, the use of synthetic spike-ins does not correspond to the physiological and biological conditions under investigation and they do not have the same effect as the housekeeping genes. Another obstacle was not having the same input of RNA/miRNA concentration across the samples collected from the patients at various time-points. Therefore, median normalization was used, which is more frequently used in next-generation sequencing $(69,70)$ and microarray (71) data normalization.

Some oncomiRs were found to be downregulated 1 week after surgical removal of the tumor $(17,20)$. It may be speculated that upregulation of oncomiRs in post-operative samples may be associated with post-operative wound healing. The present study demonstrated a decrease in these oncomiRs later at a follow-up sampling. At present, intestinal wound healing is only partially understood. It is predominantly studied in inflammatory bowel disease as well as in Crohn's disease and ulcerative colitis. During acute and chronic intestinal inflammation, immune cells such as neutrophils and macrophages induce local tissue damage by secreting tissue-degrading enzymes, reactive oxygen radicals and pro-inflammatory cytokines. The process promotes migration of myofibroblast cells to the site of the defect, arranging contractility of the wound area and the production of extracellular matrix (ECM) (72). ECM is composed of macromolecules such as proteoglycans (pathway hsa05205), non-proteoglycan polysaccharides, proteins such as collagen and elastin, and EVs. ECM also has a major function in cell adhesion (hsa04510) (73) and intestinal healing is also associated with intestinal stem cell differentiation and proliferation (hsa04550) (74,75). Notably, Hippo signalling (hsa04390) also has a role in regulating the regeneration of organs such as the liver, heart, nervous system and skin as well as intestine (76) and also prolactin (hsa04917) has been suggested to have an important role in re-epithelialization and promote wound healing (77). According to a previous study, plasma and wound fluid levels of proangiogenic proteins were elevated in patients after $\mathrm{CRC}$ resection for 3 to 5 weeks, leading to the hypothesis that the wound healing may stimulate tumour growth in residual tumour deposits (78).

The tumour microenvironment (TME) is composed of blood vessels, ECM, RNA, secreted proteins, small organelles and various populations of stromal cells (fibroblasts, adipocytes, pericytes and immune/inflammatory cells) (79). TME cells have a role in the communication between them, which is performed in autocrine, paracrine and/or endocrine ways. These cells produce growth factors and cytokines which modify molecular and cellular processes and thus alter the maturation of the TME (80).

In general, the TME participates in immune cell recruitment and activation, ECM alterations and angiogenesis, which contribute to tumour progression and wound healing (81). ECM proteins are an important component of the TME and it is well-known that changes in the composition of ECM proteins are responsible for the genesis of CRC (82). Fibrous proteins, proteoglycans, collagen and elastin as the major types of ECM proteins provide structural support and elasticity in all tissues (83). Various studies suggested that expression of high levels of proinflammatory cytokines, such as TNF produced by tumour-associated macrophages, induces neoplasm growth and invasion (84). Inflammation is involved in CRC development and progression and in post-operative intestinal wound healing. Chronic inflammation is linked to alterations in the TME through cytokines, chemokines and growth factors (85). Li et al (86) reported that elastin recombinant protein increased the proliferation of CRC epithelial cells, induced EMT, increased TNF secretion by bone marrow-derived macrophages and reduced E-cadherin in CRC epithelial cells. Inflammation is a trigger of ECM remodelling and furthermore, Li et al (86) demonstrated that proteins of ECM regulate inflammation.

In the present study, the panel of selected miRNAs was investigated in plasma of patients with CRC at the pre-operative stage, in the early post-operative period and 3 months after surgery. None of the patients received any chemotherapy at the time of follow-up sampling. Significant upregulation of miR-155-5p, miR-21-5p and miR-191-5p, and downregulation of miR-16-5p was determined. In paired follow-up samples, the most relevant upregulation was observed for miR-106a-5p and miR-16-5p and downregulation for miR-21-5p. Pathway analysis outlined their role in cancer development, but the same pathways are also involved in controlled wound healing and regeneration of intestinal epithelium $(85,87)$. Of note, the present study is limited by its sample size as well as lack of samples in the follow-up group. Furthermore, functional studies are necessary to confirm the present results in association with the pathways identified by the in silico analysis. It may be speculated that miRNA expression associated with intestinal wound healing influences the expression levels in patients' circulation (87) and the healing process should be considered in studies focusing on disease recurrence (45). For post-operative sampling, a longer interval after surgery (4-6 weeks) (88) may help in the early identification of patients with CRC recurrence in the case of latent metastases. However, more studies are necessary to confirm these results.

The selected miRNAs seemed to be promising markers for the monitoring of miRNA expression in the patients' circulation. However, the present results require confirmation in a larger cohort of patients. miRNA studies should also consider 
all of the physiological conditions that are taking place in real time in the organism and are not influenced by sample processing and technical variations. Identifying appropriate biomarkers to determine a higher risk of CRC recurrence and biomarkers to predict patient response to adjuvant treatment is a novel way to monitor and treat patients. Based on these results, it may be possible to select patients in need of more intensive monitoring as well as patients who are unlikely to benefit from adjuvant therapy.

\section{Acknowledgements}

The authors would like to thank to Professor Zora Lasabova (Department of Molecular Biology and Genomics, Jessenius Faculty of Medicine in Martin, Comenius University, Bratislava, Slovakia) for providing sample analysis and financial support.

\section{Funding}

This study was supported by the Project of Slovak Research and Development Agency (grant no. APVV-16-0066) as well as by a grant from The Ministry of Education, Science, Research and Sport of the Slovak Republic (grant no. VEGA-1/0380/18) and by the Operational Programme Integrated Infrastructure for the project 'Integrative strategy in development of personalized medicine of selected malignant tumours and its impact on quality of life' (grant no. IMTS: 313011V446), co-financed by the European Regional Development Fund.

\section{Availability of data and materials}

The datasets used and/or analyzed during the current study are available from the corresponding author on reasonable request.

\section{Authors' contributions}

Conceptualization, ZL and LL; methodology, ErK, VH and $\mathrm{ZK}$; software, $\mathrm{VH}, \mathrm{ZK}, \mathrm{MG}$; validation, $\mathrm{VH}$ and $\mathrm{ZK}$; formal analysis, VH and MG; investigation, EvK, VH, MSa, BV, PM and MSm; resources, ErK, VH, MSa, BV, PM and MSm; data curation, EvK, VH, ZK, MG and PM; writing-original draft preparation, EvK and $\mathrm{VH}$; writing - review and editing, $\mathrm{MSa}$, ErK, LL and ZL; supervision, ZL and LL; project administration, ZL; funding acquisition, ZL. VH and ErK confirm the authenticity of all the raw data. All authors have read and approved the final version of the manuscript.

\section{Ethics approval and consent to participate}

All patients were informed about this study and provided written informed consent. This research was approved by the Ethical Committee at Jessenius Faculty of Medicine in Martin, Comenius University in Bratislava (Martin, Slovakia).

\section{Patient consent for publication}

Not applicable.

\section{Competing interests}

The authors declare that they have no competing interests.

\section{References}

1. World Health Organization: International Agency For Research on Cancer: Cancer Today. Data visualization tools for exploring the global cancer burden in 2020. Available from: https://gco. iarc.fr/today/home.

2. Song M and Chan AT: Environmental factors, gut microbiota, and colorectal cancer prevention. Clin Gastroenterol Hepatol 17: 275-289, 2019

3. Dong Y, Zhou J, Zhu Y, Luo L, He T, Hu H, Liu H, Zhang Y, Luo D, Xu S, et al: Abdominal obesity and colorectal cancer risk: Systematic review and meta-analysis of prospective studies. Biosci Rep: Dec 12, 2017 (Epub ahead of print). doi: 10.1042/ BSR20170945.

4. Keum N and Giovannucci E: Global burden of colorectal cancer: Emerging trends, risk factors and prevention strategies. Nat Rev Gastroenterol Hepatol 16: 713-732, 2019.

5. Balacescu O, Sur D, Cainap C, Visan S, Cruceriu D, Manzat-Saplacan R, Muresan MS, Balacescu L, Lisencu C and Irimie A: The impact of miRNA in colorectal cancer progression and its liver metastases. Int J Mol Sci 19: 3711, 2018.

6. Riihimäki M, Hemminki A, Sundquist J and Hemminki K: Patterns of metastasis in colon and rectal cancer. Sci Rep 6: 29765, 2016.

7. Peng Y and Croce CM: The role of MicroRNAs in human cancer. Signal Transduct Target Ther 1: 15004, 2016.

8. Jin XH, Lu S and Wang AF: Expression and clinical significance of miR-4516 and miR-21-5p in serum of patients with colorectal cancer. BMC Cancer 20: 241, 2020.

9. Strubberg AM and Madison BB: MicroRNAs in the etiology of colorectal cancer: Pathways and clinical implications. Dis Model Mech 10: 197-214, 2017.

10. Jung G, Hernández-Illán E, Moreira L, Balaguer F and Goel A: Epigenetics of colorectal cancer: Biomarker and therapeutic potential. Nat Rev Gastroenterol Hepatol 17: 111-130, 2020.

11. Cojocneanu R, Braicu C, Raduly L, Jurj A, Zanoaga O, Magdo L, Irimie A, Muresan MS, Ionescu C, Grigorescu M and Berindan-Neagoe I: Plasma and tissue specific miRNA expression pattern and functional analysis associated to colorectal cancer patients. Cancers (Basel) 12: 843, 2020.

12. Ghareib AF, Mohamed RH, Abd El-Fatah AR and Saadawy SF: Assessment of serum MicroRNA-21 gene expression for diagnosis and prognosis of colorectal cancer. J Gastrointest Cancer 51: 818-823, 2020.

13. Wan TM, Iyer DN and Ng L: Roles of microRNAs as non-invasive biomarker and therapeutic target in colorectal cancer. Histol Histopathol 35: 225-237, 2020.

14. Locker GY, Hamilton S, Harris J, Jessup JM, Kemeny N, Macdonald JS, Somerfield MR, Hayes DF and Bast RC Jr; ASCO: ASCO 2006 update of recommendations for the use of tumor markers in gastrointestinal cancer. J Clin Oncol 24: 5313-5327, 2006.

15. Duffy MJ, van Dalen A, Haglund C, Hansson L, Holinski-Feder E, Klapdor R, Lamerz R, Peltomaki P, Sturgeon C and Topolcan O: Tumour markers in colorectal cancer: European Group on Tumour Markers (EGTM) guidelines for clinical use. Eur J Cancer 43: 1348-1360, 2007.

16. Tan E, Gouvas N, Nicholls RJ, Ziprin P, Xynos E and Tekkis PP: Diagnostic precision of carcinoembryonic antigen in the detection of recurrence of colorectal cancer. Surg Oncol 18: 15-24, 2009.

17. Pesta M, Kucera R, Topolcan O, et al: Plasma microRNA levels combined with CEA and CA19-9 in the follow-up of colorectal cancer patients. Cancers (Basel) 11: E864, 2019.

18. Iorio MV and Croce CM: microRNA involvement in human cancer. Carcinogenesis 33: 1126-1133, 2012.

19. Piccinini AM and Midwood KS: Endogenous control of immunity against infection: Tenascin-C regulates TLR4-mediated inflammation via microRNA-155. Cell Rep 2: 914-926, 2012.

20. Tili E, Michaille JJ and Croce CM: MicroRNAs play a central role in molecular dysfunctions linking inflammation with cancer. Immunol Rev 253: 167-184, 2013.

21. Ristau J, Staffa J, Schrotz-King P, Gigic B, Makar KW, Hoffmeister M, Brenner H, Ulrich A, Schneider M, Ulrich CM and Habermann N: Suitability of circulating miRNAs as potential prognostic markers in colorectal cancer. Cancer Epidemiol Biomark Prev 23: 2632-2637, 2014. 
22. Chen B, Xia Z, Deng YN, Yang Y, Zhang P, Zhu H, Xu N and Liang S: Emerging microRNA biomarkers for colorectal cancer diagnosis and prognosis. Open Biol 9: 180212.

23. Rapado-González Ó, Álvarez-Castro A, López-López R, Iglesias-Canle J, Suárez-Cunqueiro MM and Muinelo-Romay L: Circulating microRNAs as promising biomarkers in colorectal cancer. Cancers (Basel) 11: 898, 2019.

24. Tsukamoto M, Iinuma H, Yagi T, Matsuda $\mathrm{K}$ and Hashiguchi $\mathrm{Y}$ : Circulating exosomal MicroRNA-21 as a biomarker in each tumor stage of colorectal cancer. Oncology 92: 360-370, 2017.

25. Danese E, Minicozzi AM, Benati M, Paviati E, Lima-Oliveira G, Gusella M, Pasini F, Salvagno GL, Montagnana M and Lippi G: Reference miRNAs for colorectal cancer: Analysis and verification of current data. Sci Rep 7: 8413, 2017.

26. Chen J, Wang W, Zhang Y, Chen Y and Hu T: Predicting distant metastasis and chemoresistance using plasma miRNAs. Med Oncol Northwood Lond Engl 31: 799, 2014

27. Luu-The V, Paquet N, Calvo E and Cumps J: Improved real-time RT-PCR method for high-throughput measurements using second derivative calculation and double correction. Biotechniques 38 287-293, 2005

28. R Core Team: R: A Language and Environment for Statistical Computing. R Foundation for Statistical Computing, Vienna, Austria, 2018.

29. Livak KJ and Schmittgen TD: Analysis of relative gene expression data using real-time quantitative PCR and the 2(-Delta Delta C(T)) method. Methods 25: 402-408, 2001

30. Vlachos IS, Zagganas K, Paraskevopoulou MD, Georgakilas G, Karagkouni D, Vergoulis T, Dalamagas T and Hatzigeorgiou AG: DIANA-miRPath v3.0: Deciphering microRNA function with experimental support. Nucleic Acids Res 43: W460-W466, 2015.

31. Kirschner MB, Kao SC, Edelman JJ, Armstrong NJ, Vallely MP, Zandwijk N van and Reid G: Haemolysis during sample preparation alters microRNA content of plasma. PLoS One 6: e24145, 2011

32. Goni R, García P and Foissac S: The qPCR data statistical analysis. 9, 2009

33. Chen WY, Zhao XJ, Yu ZF, Hu FL, Liu YP, Cui BB, Dong XS and Zhao YS: The potential of plasma miRNAs for diagnosis and risk estimation of colorectal cancer. Int J Clin Exp Pathol 8 7092-7101, 2015.

34. Li J, Liu Y, Wang C, Deng T, Liang H, Wang Y, Huang D, Fan Q, Wang X, Ning T, et al: Serum miRNA expression profile as a prognostic biomarker of stage II/III colorectal adenocarcinoma. Sci Rep 5: 12921, 2015

35. He Y, Wang G, Zhang L, Zhai C, Zhang J, Zhao X, Jiang X and Zhao Z: Biological effects and clinical characteristics of microRNA-106a in human colorectal cancer. Oncol Lett 14 830-836, 2017.

36. Díaz R, Silva J, García JM, Lorenzo Y, García V, Peña C Rodríguez R, Muñoz C, García F, Bonilla F and Domínguez G: Deregulated expression of miR-106a predicts survival in human colon cancer patients. Genes Chromosomes Cancer 47: 794-802, 2008.

37. Yue B, Sun B, Liu C, Zhao S, Zhang D, Yu F and Yan D: Long non-coding RNA Fer-1-like protein 4 suppresses oncogenesis and exhibits prognostic value by associating with miR-106a-5p in colon cancer. Cancer Sci 106: 1323-1332, 2015.

38. Peng Q, Shen Y, Zhao P, Cheng M, Zhu Y and Xu B: Biomarker roles identification of miR-106 family for predicting the risk and poor survival of colorectal cancer. BMC Cancer 20: 506 , 2020.

39. Wang C, Wang J, Liu H and Fu Z: Tumor suppressor DLC-1 induces apoptosis and inhibits the growth and invasion of colon cancer cells through the Wnt/ $\beta$-catenin signaling pathway. Oncol Rep 31: 2270-2278, 2014

40. Cao H, Huang S, Liu A and Chen Z: Up-regulated expression of miR-155 in human colonic cancer. J Cancer Res Ther 14 604-607, 2018

41. Liu N, Jiang F, Han XY, Li M, Chen WJ, Liu QC, Liao CX and Lv YF: MiRNA-155 promotes the invasion of colorectal cancer SW-480 cells through regulating the Wnt/ $\beta$-catenin. Eur Rev Med Pharmacol Sci 22: 101-109, 2018.

42. Qu A, Du L, Yang Y, Liu H, Li J, Wang L, Liu Y, Dong Z, Zhang X, Jiang X, et al: Hypoxia-inducible MiR-210 is an independent prognostic factor and contributes to metastasis in colorectal cancer. PLoS One 9: e90952, 2014

43. Sabry D, El-Deek SEM, Maher M, El-Baz MAH, El-Bader HM, Amer E, Hassan EA, Fathy W and El-Deek HEM: Role of miRNA-210, miRNA-21 and miRNA-126 as diagnostic biomarkers in colorectal carcinoma: Impact of HIF-1 $\alpha$-VEGF signaling pathway. Mol Cell Biochem 454: 177-189, 2019.
44. Ullmann P, Qureshi-Baig K, Rodriguez F, Ginolhac A, Nonnenmacher Y, Ternes D, Weiler J, Gäbler K, Bahlawane C, Hiller K, et al: Hypoxia-responsive miR-210 promotes self-renewal capacity of colon tumor-initiating cells by repressing ISCU and by inducing lactate production. Oncotarget 7: 65454-65470, 2016.

45. Jung EJ, Santarpia L, Kim J, Esteva FJ, Moretti E, Buzdar AU, Di Leo A, Le XF, Bast RC Jr, Park ST, et al: Plasma microRNA 210 levels correlate with sensitivity to trastuzumab and tumor presence in breast cancer patients. Cancer 118: 2603-2614, 2012.

46. Wan J, Xia L, Xu W and Lu N: Expression and function of miR-155 in diseases of the gastrointestinal Tract. Int J Mol Sci 17: 709, 2016

47. Volinia S, Calin GA, Liu CG, Ambs S, Cimmino A, Petrocca F, Visone R, Iorio M, Roldo C, Ferracin M, et al: A microRNA expression signature of human solid tumors defines cancer gene targets. Proc Natl Acad Sci USA 103: 2257-2261, 2006.

48. Rath T, Billmeier U, Waldner MJ, Atreya R and Neurath MF: From physiology to disease and targeted therapy: Interleukin-6 in inflammation and inflammation-associated carcinogenesis. Arch Toxicol 89: 541-554, 2015.

49. Svrcek M, El-Murr N, Wanherdrick K, Dumont S, Beaugerie L, Cosnes J, Colombel JF, Tiret E, Fléjou JF, Lesuffleur T and Duval A: Overexpression of microRNAs-155 and 21 targeting mismatch repair proteins in inflammatory bowel diseases. Carcinogenesis 34: 828-834, 2013.

50. Ulivi P, Canale M, Passardi A, Marisi G, Valgiusti M, Frassineti GL, Calistri D, Amadori D and Scarpi E: Circulating plasma levels of miR-20b, miR-29b and miR-155 as predictors of bevacizumab efficacy in patients with metastatic colorectal cancer. Int J Mol Sci 19: 307, 2018.

51. You C, Jin L, Xu Q, Shen B, Jiao X and Huang X: Expression of miR-21 and miR-138 in colon cancer and its effect on cell proliferation and prognosis. Oncol Lett 17: 2271-2277, 2019.

52. Mima K, Nishihara R, Yang J, Dou R, Masugi Y, Shi Y, da Silva A, Cao Y, Song M, Nowak J, et al: MicroRNA MIR21 (miR-21) and PTGS2 expression in colorectal cancer and patient survival. Clin Cancer Res 22: 3841-3848, 2016.

53. Inamura $\mathrm{K}$ and Ishikawa $\mathrm{Y}$ : MicroRNA in lung cancer: Nove biomarkers and potential tools for treatment. J Clin Med 5: 36, 2016.

54. Komatsu S, Ichikawa D, Takeshita H, Tsujiura M, Morimura R, Nagata H, Kosuga T, Iitaka D, Konishi H, Shiozaki A, et al: Circulating microRNAs in plasma of patients with oesophageal squamous cell carcinoma. Br J Cancer 105: 104-111, 2011.

55. Saxena A, Tammali R, Ramana KV and Srivastava SK: Aldose reductase inhibition prevents colon cancer growth by restoring phosphatase and tensin homolog through modulation of miR-21 and FOXO3a. Antioxid Redox Signal 18: 1249-1262, 2013.

56. Hong Z, Feng Z, Sai Z and Tao S: PER3, a novel target of miR-103, plays a suppressive role in colorectal cancer in vitro. BMB Rep 47: 500-505, 2014

57. Zheng YB, Xiao K, Xiao GC, Tong SL, Ding Y, Wang QS, Li SB and Hao ZN: MicroRNA-103 promotes tumor growth and metastasis in colorectal cancer by directly targeting LATS2. Oncol Lett 12: 2194-2200, 2016.

58. Wang DS, Zhong B, Zhang M-S and Gao Y: Upregulation of serum miR-103 predicts unfavorable prognosis in patients with colorectal cancer. Eur Rev Med Pharmacol Sci 22: 4518-4523, 2018.

59. Xu W, Ji J, Xu Y, Liu Y, Shi L, Liu Y, Lu X, Zhao Y, Luo F, Wang B, et al: MicroRNA-191, by promoting the EMT and increasing CSC-like properties, is involved in neoplastic and metastatic properties of transformed human bronchial epithelial cells. Mol Carcinog 54 (Suppl 1): E148-E161, 2015.

60. Zhang XF, Li KK, Gao L, Li SZ, Chen K, Zhang JB, Wang D, Tu RF, Zhang JX, Tao KX, et al: miR-191 promotes tumorigenesis of human colorectal cancer through targeting $\mathrm{C} / \mathrm{EBP} \beta$. Oncotarget 6: 4144-4158, 2015.

61. Qin S,Zhu Y,Ai F, Li Y, Bai B, Yao W and Dong L: MicroRNA-191 correlates with poor prognosis of colorectal carcinoma and plays multiple roles by targeting tissue inhibitor of metalloprotease 3 . Neoplasma 61: 27-34, 2014.

62. Milanesi E, Dobre M, Bucuroiu AI, Herlea V, Manuc TE, Salvi A, De Petro G, Manuc M and Becheanu G: miRNAs-based molecular signature for KRAS mutated and wild type colorectal cancer: An explorative study. J Immunol Res 2020: 4927120, 2020.

63. Chen XY, Zhang J, Hou LD, Zhang R, Chen W, Fan HN, Huang YX, Liu $\mathrm{H}$ and Zhu JS: Upregulation of PD-L1 predicts poor prognosis and is associated with miR-191-5p dysregulation in colon adenocarcinoma. Int J Immunopathol Pharmacol 32: 2058738418790318,2018 
64. Qian J, Jiang B, Li M, Chen J and Fang M: Prognostic significance of microRNA-16 expression in human colorectal cancer. World J Surg 37: 2944-2949, 2013.

65. Xiao G, Tang H, Wei W, Li J, Ji L and Ge J: Aberrant expression of MicroRNA-15a and MicroRNA-16 synergistically associates with tumor progression and prognosis in patients with colorectal cancer. Gastroenterol Res Pract 2014: 364549, 2014.

66. Ma Q, Wang X, Li Z, Li B, Ma F, Peng L, Zhang Y, Xu A and Jiang B: microRNA-16 represses colorectal cancer cell growth in vitro by regulating the p53/survivin signaling pathway. Oncol Rep 29: 1652-1658, 2013.

67. Diamantopoulos MA, Kontos CK, Kerimis D, Papadopoulos IN and Scorilas A: Upregulated miR-16 expression is an independent indicator of relapse and poor overall survival of colorectal adenocarcinoma patients. Clin Chem Lab Med 55: 737-747, 2017.

68. Ostenfeld MS, Jensen SG, Jeppesen DK, Christensen LL, Thorsen SB, Stenvang J, Hvam ML, Thomsen A, Mouritzen P, Rasmussen $\mathrm{MH}$, et al: miRNA profiling of circulating EpCAM(+) extracellular vesicles: Promising biomarkers of colorectal cancer. J Extracell Vesicles 5: 31488, 2016.

69. Evans C, Hardin J and Stoebel D: Selecting between-sample RNA-Seq normalization methods from the perspective of their assumptions. Brief Bioinform 19: 776-792, 2018

70. Dillies MA, Rau A, Aubert J,Hennequet-Antier C, Jeanmougin M, Servant N, Keime C, Marot G, Castel D, Estelle J, et al: A comprehensive evaluation of normalization methods for Illumina high-throughput RNA sequencing data analysis. Brief Bioinform 14: 671-683, 2013.

71. Fundel K, Haag J, Gebhard PM, Zimmer R and Aigner T: Normalization strategies for mRNA expression data in cartilage research. Osteoarthritis Cartilage 16: 947-955, 2008.

72. Rieder F, Brenmoehl J, Leeb S, Schölmerich J and Rogler G: Wound healing and fibrosis in intestinal disease. Gut 56: 130-139, 2007.

73. Theocharis AD, Skandalis SS, Gialeli C and Karamanos NK: Extracellular matrix structure. Adv Drug Deliv Rev 97: 4-27, 2016.

74. Howell JC and Wells JM: Generating intestinal tissue from stem cells: Potential for research and therapy. Regen Med 6: 743-755, 2011.

75. Xue X and Falcon DM: The role of immune cells and cytokines in intestinal wound healing. Int J Mol Sci 20: 6097, 2019.

76. Wang Y, Yu A and Yu FX: The Hippo pathway in tissue homeostasis and regeneration. Protein Cell 8: 349-359, 2017.
77. Wang H, Li X, Lu J, Jones $\mathrm{P}$ and $\mathrm{Xu} \mathrm{W}$ : Prolactin may serve as a regulator to promote vocal fold wound healing. Biosci Rep 40: BSR20200467, 2020.

78. Shantha Kumara H, Yan X-H, Pettke E, Cekic V, Gandhi ND, Bellini GA and Whelan RL: Plasma and wound fluid levels of eight proangiogenic proteins are elevated after colorectal resection. World J Gastrointest Oncol 11: 470-488, 2019.

79. Mizuno R, Kawada K, Itatani Y, Ogawa R, Kiyasu Y and Sakai Y: The role of tumor-associated neutrophils in colorectal cancer. Int J Mol Sci 20: 529, 2019.

80. Roma-Rodrigues C, Mendes R, Baptista PV and Fernandes AR: Targeting tumor microenvironment for cancer therapy. Int J Mol Sci 20: 840, 2019.

81. Kasprzak A: The role of tumor microenvironment cells in colorectal cancer (CRC) cachexia. Int J Mol Sci 22: 1565, 2021.

82. Bonnans C, Chou J and Werb Z: Remodelling the extracellular matrix in development and disease. Nat Rev Mol Cell Biol 15: 786-801, 2014.

83. Salesse S, Odoul L, Chazée L, Garbar C, Duca L, Martiny L, Mahmoudi R and Debelle L: Elastin molecular aging promotes MDA-MB-231 breast cancer cell invasiveness. FEBS Open Bio 8: 1395-1404, 2018.

84. Greten FR and Grivennikov SI: Inflammation and Cancer: Triggers, Mechanisms, and Consequences. Immunity 51: 27-41, 2019.

85. Triner D and Shah YM: Hypoxia-inducible factors: A central link between inflammation and cancer. J Clin Invest 126: 3689-3698, 2016.

86. Li J, Xu X, Jiang Y, Hansbro NG, Hansbro PM, Xu J and Liu G: Elastin is a key factor of tumor development in colorectal cancer. BMC Cancer 20: 217, 2020.

87. Sommer K, Wiendl M, Müller TM, Heidbreder K, Voskens C, Neurath MF and Zundler S: Intestinal mucosal wound healing and barrier integrity in IBD-crosstalk and trafficking of cellular players. Front Med (Lausanne) 8: 643973, 2021.

88. Monnet E and Smeak DD: Gastrointestinal healing. In Gastrointestinal Surgical Techniques in Small Animals. Monnet E and Smeak DD (eds). Wiley Blackwell, Hoboken, NJ, USA, pp 1-8, 2020.

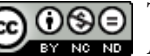

This work is licensed under a Creative Commons Attribution-NonCommercial-NoDerivatives 4.0 International (CC BY-NC-ND 4.0) License. 